Radial and Nonradial Pulsations as Probes of Stellar Physics

ASP Conference Series, Vol. 259, 2002

C. Aerts, T.R. Bedding, \& J. Christensen-Dalsgaard, eds.

\title{
SX Phe Variables in Globular Clusters
}

\author{
E. Rodríguez, M.J. López-González \\ Instituto de Astrofísica de Andalucía, CSIC, P.O. Box 3004, E-18080 \\ Granada, Spain
}

\begin{abstract}
We have compiled an extensive and up-dated list of SX Phetype pulsators in globular clusters. In addition, SX Phe variables discovered in the Carina dwarf galaxy and other ones probably belonging to the Sagittarius dwarf galaxy are included. This catalogue is intended to be an up-dated list of all the SX Phe stars, in globular clusters and galaxies, known until now. The stars and their most significant parameters are listed together with references to studies of individual stars and notes for a number of variables. In addition, the references on the discovery for each variable and sources for photometry are also listed. Some relevant distributions are also analysed. The analysis of metal abundances and mean periods shows that both parameters are correlated in the sense that the periods of the variables are longer as the metallicity of the stellar system is higher.
\end{abstract}

\section{Discussion}

Very few SX Phe variables are known in the field. Recent reviews on $\delta$ Sct and SX Phe field variables are available in the literature (Breger, 2000; Rodriguez et al., 2000 and references therein). The fact that $\delta$ Sct and SX Phe variables are different groups was confirmed by the discovery of three SX Phe-type pulsators among the blue stragglers population of the globular cluster $\omega$ Cen (NJL220, NJL79 and E39). The SX Phe-type variables are not yet fully explained by the stellar evolution theory. The connection between these stars and the bluestraggler phenomenon has been analysed by different authors. A number of reviews are also available in the bibliography (Nemec et al., 1994; McNamara, 1995, 1997).

Recently, a lot of these variables are being discovered in globular clusters. In this respect, the contributions from the OGLE side-projects are of relevant significance. In addition, a large number of SX Phe pulsators have been found in the Carina dwarf spheroidal galaxy and three new members probably belonging to the Sagittarius dwarf galaxy. The main aim of this work is to present an extensive and up-dated list of SX Phe variables in globular clusters and galaxies, covering the information published until now. The full list together with references, notes and sources are accessible in electronic form and can also be requested from the authors. All these variables were discovered very recently (later than the year 1980) and there is also an enormous increase during the last 
few years. In fact, $70 \%$ of the full sample were discovered since 1996 and nearly $90 \%$ were discovered during the last decade.

The variables show visual amplitudes from a few hundredths of a magnitude to several tenths, however the number of variables increases as the amplitude is decreasing. In fact, we have about $45 \%$ of the SX Phe stars pulsating with amplitudes smaller than $0 .^{m} 1$ and $25 \%$ with amplitudes smaller than $0 .^{m} 05$. This suggests that we cannot exclude the possibility that many of the apparently nonvarying stars in the SX Phe region vary but with undetectable amplitudes.

Moreover, nearly all of these stars have very short periods, ranging from $0 .{ }^{d} 025$ ( 0.6 hours) to $0 .{ }^{d} 075$ ( 1.8 hours). The variable with the shortest period in our list is V10 in the globular cluster NGC 6397 with $\mathrm{P}=43$ min (Kaluzny, 1997) and there are only 3 stars with periods longer than $0 .{ }^{d} 1$. This is quite different to that shown by the $\delta$ Sct-type pulsators, where the periods can be much longer $\left(<0 .{ }^{d} 3\right)$. Moreover, the variables with longer periods $\left(0 .{ }^{d} 050-0.0^{d} 075\right)$ that seem to have larger visual amplitudes than those with shorter periods $\left(0 .{ }^{d} 025-0 .{ }^{d} 050\right)$.

On the other hand, the distribution of the variables versus the visual magnitude shows that the majority of the stars (86) have visual magnitudes between $16 .^{m} 0$ to $18 .^{m} 0$ and all the variables fainter than $22 .^{m} 0$ belong to the Carina dwarf galaxy. It seems there is a selection effect in our catalogue in the sense that the amplitude is larger when the variable is fainter, that is, only the largest amplitude faint stars can be detected to be variables.

Finally, if we plot the metallicity versus mean period for each cluster we can find a correlation between both parameters in the sense that the periods of the variables are longer when the metallicity of the system is higher. The scatter is much smaller when only the fundamental periods are plotted. Following the suggestion of McNamara $(1995,1997)$ we can assume that the SX Phe variables with large amplitudes are pulsating in the fundamental mode while those variables with $\Delta V \leq 0,{ }^{m} 20$ are first-overtone pulsators. The fundamental periods of each variable belonging to this second group can be calculated assuming $\mathrm{P}_{1} / \mathrm{P}_{0}=0.778$. Thus, an improved correlation between $[\mathrm{Me} / \mathrm{H}]$ and $\log \mathrm{P}_{0}$ is found.

\section{References}

Breger, M. 2000, in ASP Conf. Ser., Vol. 210, Delta Scuti and Related Stars, eds. M. Breger \& M. Montgomery, (San Francisco ASP), I

Kaluzny, J. 1997, A\&AS, 122, 1

McNamara, D.H. 1995, AJ, 109, 1751

McNamara, D.H. 1997, PASP, 109, 1221

Nemec, J.M., Nemec, A.F.L., \& Lutz, T.E. 1994, AJ, 108, 222

Rodríguez, E., López-González, M.J., \& López de Coca, P. 2000, A\&AS, 144, 469 